\title{
Statistical Distributions of Electron Avalanches and Streamers
}

\author{
T. Ficker
}

A new theoretical concept of fractal multiplication of electron avalanches has resulted in forming a generalized distribution function whose multiparameter character has been subjected to detailed discussion.

Keywords: fractal multiplication of electron avalanches, Furry and Pareto statistics, fractal dimension.

\section{Introduction}

So far it has been believed [1] that electron populations $n$ of avalanches crossing a discharge gap $d$ in a homogeneous electric field are governed by Furry statistics [2-3]

$w_{0}(n, d)=\frac{1}{\bar{n}}\left[1-\frac{1}{\bar{n}}\right]^{n-1} \stackrel{\bar{n} \gg 1}{\longrightarrow} \frac{1}{\bar{n}} \exp \left(-\frac{n}{\bar{n}}\right)$,

$\bar{n}=\exp \left[\int_{0}^{d} \alpha\left(x^{\prime}\right) \mathrm{d} x^{\prime}\right]$

where $w_{0}$ and $\alpha$ are the probability density function and the first Townsend ionization coefficient, respectively. The validity of this law used to be accepted [1] independently of the size of the avalanches, i.e., regardless of the magnitude of their mean electron content $\bar{n}$, in spite of the fact that there were strong indications [1], [4-5] showing different statistical behavior. Especially with highly populated avalanches $\bar{n}>10^{5}$, clear deviations from the Furry law (1) were often observed [4-5]. It has been illustrated many times [6-12] that the population statistics of such highly populated avalanches and streamers obey Pareto (fractal) statistics

$$
w_{1}(n, d)=a \cdot \boldsymbol{n}^{-(1+D)}
$$

where $a$ is a constant and $D$ is the so-called fractal dimension [13]. Convincing examples of different statistical behavior of highly and lowly populated avalanches can be found in the earlier work of Richter [5]. He measured population statistics in ether under discharge conditions that were favorable for creating a mixture of pre-streamer and streamer avalanches, with a majority of the latter. Such highly populated avalanches $\left(n>10^{8}\right)$ provided distribution functions with a very deep bending in the semilogarithmic co-ordinate system, where the population statistics of avalanches should show linear behavior in accordance with the Furry law. When analyzing Richter's curves in the bilogarithmic system, one can easily recognize two neighboring different regions (see Fig. 1). There is a longer linear part and a shorter non-linear (bent) part. The bending of the latter corresponds to the exponential Furry behavior typical for less populated avalanches, whereas the linear part represents the Pareto behavior characteristic for highly populated avalanches and streamers. This figure clearly illustrates that the exponential and power fitting functions in the Furry and Pareto regions, respectively, represent a good choice among possible analytical candidates, since both the fits follow the experimental data well.

Another instructive example showing how well the Pareto power function represents the experimental data is given in Fig. 2. In this case, avalanches were detected across a resistance as short voltage pulses with random heights $u$. (The resistance $(R=100 \mathrm{k} \Omega$ ) was connected in series to the discharge gap (C) so that the two components formed a classical RC-circuit - for more details see [14].) Since the voltage pulses $u$ were not calibrated against the number of electrons $n$, the resultant distribution curves $w_{1}$ are dependent on $u$ instead of $n$. Assuming linear proportionality $u \approx c \cdot n$, the curve $w_{1}(u)=c_{0} \cdot u^{-(D+1)}$ will preserve the same shape as $w_{1}(n)$, i.e. they will both possess the same slope $-(1+D)$.

Recently a new statistical pattern has been developed [14]

$$
\begin{aligned}
w(n, d) & =\frac{G}{\bar{n}_{d}} \sum_{j=0}^{J}(\bar{K} \cdot \bar{N})^{j} \cdot\left[1-\frac{\bar{N}^{j}}{\bar{n}_{d}}\right]^{n-1} \\
& \approx \frac{G}{\bar{n}_{d}} \sum_{j=0}^{J}(\bar{K} \cdot \bar{N})^{j} \cdot \exp \left(-\frac{n \cdot \bar{N}^{j}}{\bar{n}_{d}}\right),
\end{aligned}
$$

$w(n, d)=G \cdot F(n, d), \quad J=\frac{d}{\bar{\Delta}}-1$,

$\bar{N}>1, \quad \bar{K} \geq 1, \quad D=\frac{\ln \bar{K}}{\ln \bar{N}}$.

Pattern (3) unifies both Furry (1) and Pareto (2) statistics into a single analytical form. For example, if $J=0$, the Furry distribution results from (3), whereas for $J>0$ a superposition of Furry/exponential functions creates Pareto behavior, i.e. the linear section on the graph $w(n, d)$ plotted in bilogarithmic co-ordinates, as shown in Fig. 1. In addition, a rigorous mathematical proof was presented in Ref. [14], showing equivalence between statistical forms (2) and (3).

In order to explain the meanings of parameters $\mathrm{G}, \bar{K}, \bar{N}$, and $\bar{n}_{d}$ used in generalized analytical form (3), it is necessary 


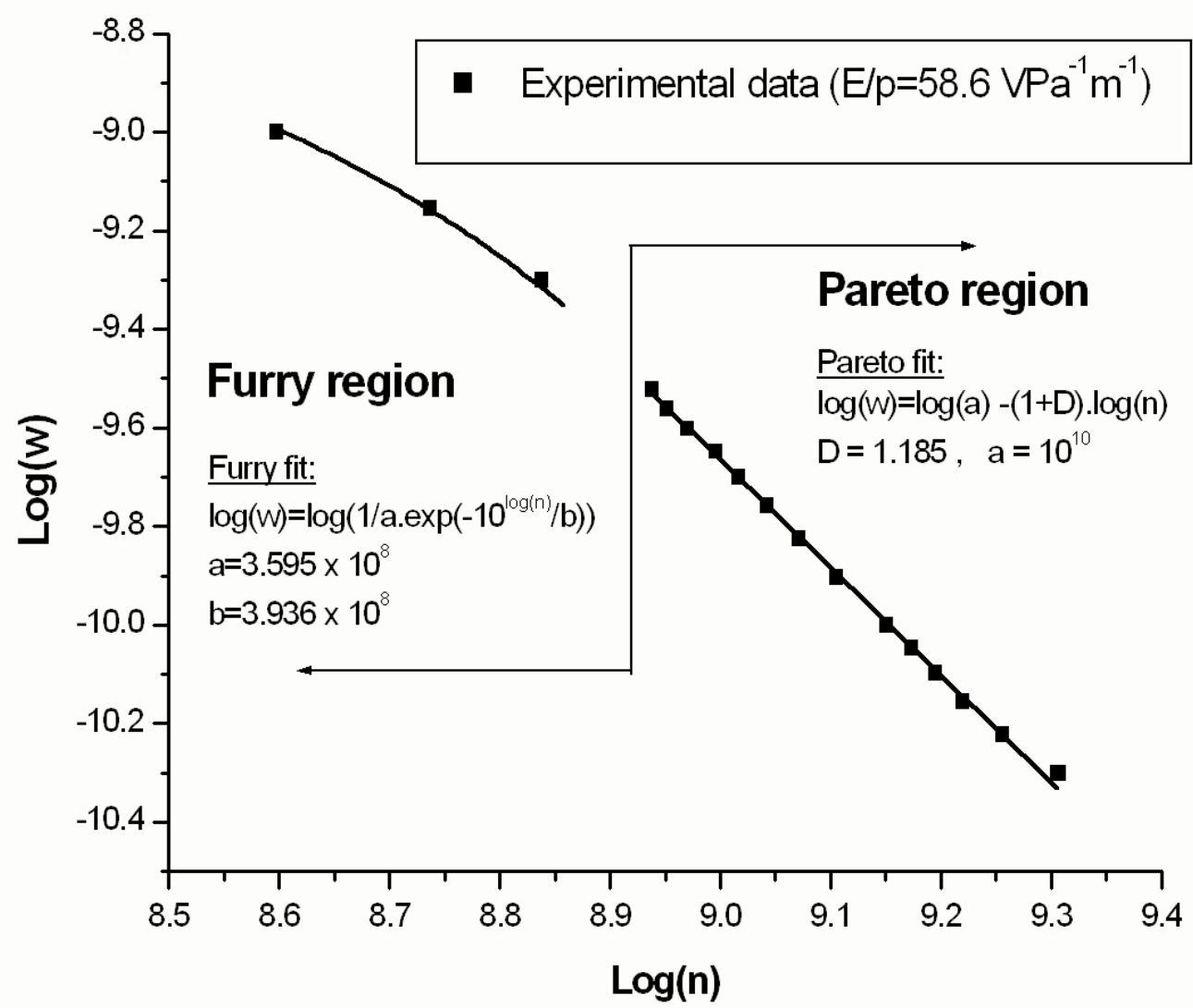

Fig. 1: Population statistics of a mixture of lowly and highly populated avalanches. Statistical relevancy: Furry fit $R^{2} \rightarrow 0.9975$ and $\chi^{2} \rightarrow 0.00018$, Pareto fit $R^{2} \rightarrow 0.998$ and $\chi^{2} \rightarrow 0.0001$. Data taken from [5].

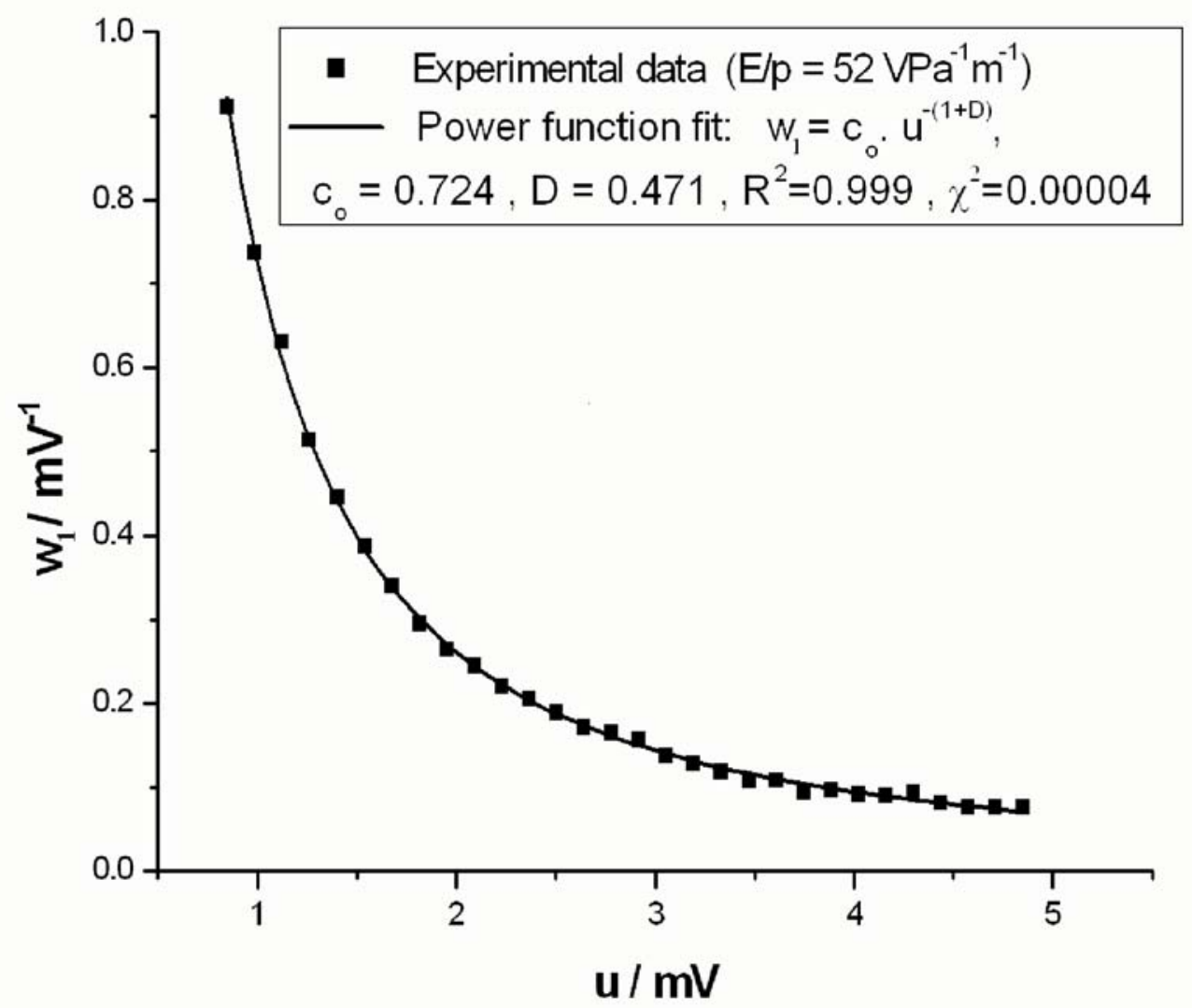

Fig. 2: Avalanche statistics (voltage pulses) in air at normal laboratory conditions. After [14]. 


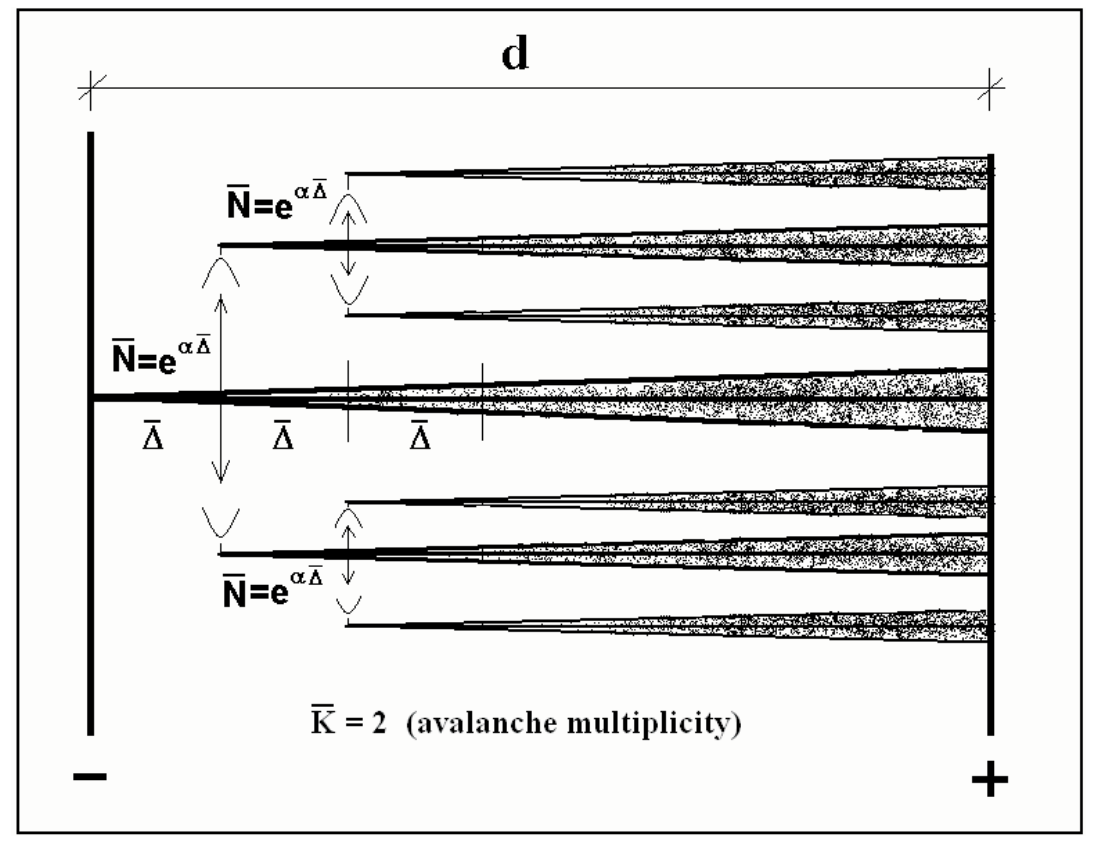

Fig. 3: Scheme of fractal avalanche multiplication. After [14].

to make a brief description of the scenario of fractal avalanche multiplication (Fig. 3). Let us consider a parent avalanche started at the cathode. After crossing a critical distance $\bar{\Delta}$, it may gather a certain number of electrons $\bar{N}=\exp (\alpha \bar{\Delta})(\alpha$ is the first Townsend ionization coefficient), and the uv-radiation associated with collisional ionization may initiate $\bar{K}$ new displaced avalanches ( $\bar{K}$ is the so-called multiplicity). The displaced avalanches continue their own independent tracks, and after passing a distance $\bar{\Delta}$ they may (or may not) generate $\bar{K}^{2}$ new displaced avalanches of the second generation. In general, the $j$-th generation of displaced avalanches contains $\bar{K}^{j}$ avalanches and the highest generation $J$ is limited by the length $d$ of the discharge gap, critical distance $\bar{\Delta}$ and the value of $\alpha$, i.e. $J=d / \bar{\Delta}-1$. The populations of displaced avalanches of the $j$-th generation that reach the anode can easily be expressed: $n_{d, j}=\exp [\alpha(d-j \bar{\Delta})]$. The term $\bar{n}_{d}=\bar{n}_{d, 0}$ is a population of the parent avalanche $(j=0)$. Each generation has its own distribution function

$$
w_{d, j}(n)=\frac{(\bar{K} \cdot \bar{N})^{j}}{\bar{n}_{d}} \cdot\left[\frac{1-\bar{N}^{j}}{\bar{n}_{d}}\right]^{n-1}
$$

and their sum over all generations $(j=0,1, \ldots, J)$ leads to statistical pattern (3) - more details can be found in Ref. [14]. The symbol $G$ represents the normalization constant. Since the process of fractal avalanche multiplication is highly stochastic, average values $\bar{K}, \bar{N}, \bar{\Delta}$ and $\bar{n}_{d}$ are used.

Creation of additional smaller avalanches inside the discharge gap is a rather different process from that of branching with streamers. While multiplication of avalanches results in creating a set of separate avalanches, streamer branching leads to a connected network of plasma channels. A common item of these two processes may be photoionization. Photoionization is the "driving force" necessary for streamer propagation, but in the case of avalanches it represents one of the possible creation mechanisms. However, streamer branching, as described by recent research papers [15-18], seems to be a complex process. Kulikovski [15] interpreted streamer branching as an instability that transforms the non-standard streamer into a number of standard streamers. Pancheshnyi [16] described the effects of streamer branching on the basis of background ionization and photoionization. Arrayas et al. [17] described the splitting at the streamer tip as a Laplacian instability. Montijn, Ebert and Hundsdorfer [18] compared this instability to the branching instability of fluid interfaces in viscous fingering. There are many other works that numerically simulate propagation and branching of streamers. Nevertheless, to our knowledge there is no work that numerically simulates fractal multiplication of avalanches as a basis for explaining the Pareto behavior of avalanche population statistics, though existing numerical models of streamer growth could determine these statistics, including avalanche multiplication.

The present paper focuses on a recently published statistical model [14] concerning populations of electron avalanches that undergo fractal multiplication within the discharge gap. The discussion focuses on the model parameters and their connections with physical processes underlying the phenomenon of fractal avalanche multiplication. The discussion ex- 
plains in detail the role of each particular parameter, and provides a deeper insight into the model operation.

\section{Discussion of model parameters}

The probability density function (3) has the character of a fitting function containing four parameters $J, \bar{K}, \bar{N}$ and $\bar{n}_{d}$ which, together with dimension $D$, deserve further discussion and explanation.

\subsection{Parameter $\bar{n}_{d}$}

This parameter represents an average number of electrons $\bar{n}_{d}=\exp (\alpha d)$ in the parent avalanche. For a given discharge gap, gas content and physical conditions, the value of $\bar{n}_{d}$ is a fixed number.

However, from the viewpoint of fractal theory $\bar{n}_{d}$ cannot be considered as a fixed reference population scale that will "anchor" all remaining avalanche components (displaced avalanches), since each generation ( $j$ ) of displaced avalanches has its own scale $\left(\bar{n}_{d} / \bar{N}^{j}\right)$ and all these population scales are as important as $\bar{n}_{d}$. Thus, the statistical set of all avalanches is a mixture of many mutually different but equally important population scales, none of which stands for a unique, basic scale defining a reference. This is one of the basic properties of all fractal objects. Now that the principle of the lost reference scale has been mentioned, it is clear that it has no sense to change the only parameter $\bar{n}_{d}$ at fixed others to model the transition from Furry statistics to Pareto statistics. The only item that will be influenced by changing $\bar{n}_{d}$ is the position of the linear section on the graph $\log (w)$ versus $\log (n)$. But if a complete set of avalanches is considered, i.e. $n \in(0, \infty)$, $\bar{n}_{d} \rightarrow \infty$ (for $d \rightarrow \infty$ ), the linear section will be infinitely long and there will be no need to change $\bar{n}_{d}$. In such a case the parameter $\bar{n}_{d}$ will lose its analytical role, which consists in shifting the linear section along the graph when an incomplete avalanche set is fitted. According to the adopted statistical concept, it is not the value of $\bar{n}_{d}$ that governs the transition between Furry and Pareto statistics. The high values of $\bar{n}_{d}$ that can be observed when such a transition occurs seem to be only an accompanying effect (not a primary effect).

\subsection{Parameter $\bar{N}$}

The value of this parameter is intimately connected with the critical distance $\bar{\Delta}$ at which the primary avalanche may generate the first displaced avalanche, i.e. $\bar{N}=\exp (\alpha \cdot \bar{\Delta})$. In other words: the average distance $\bar{\Delta}$ is necessary for the primary avalanche to assemble a certain number of electrons $\bar{N}$ that are capable of generating sufficient uv radiation (formation of photon sources) to facilitate the creation of displaced avalanches. It is assumed that the effective ionization length $\bar{\lambda}$ [15], [19], which is passed by photons prior to their absorption (photoionization events), is independent of $\bar{\Delta}$. (The photoionization process is assumed to be effective in the case of molecules that have been excited to their higher energy states in previous collisions with electrons. The different values of the first ionization potentials of $\mathrm{N}_{2}$ and $\mathrm{O}_{2}$ molecules in air make this process still easier.) Although the values of these two parameters can be arbitrarily different, both the processes, i.e. the appearance of the critical population $\bar{N}$ and the appearance of the first displaced avalanche, are almost synchronous, due to the very high speed of the photons. However, it should be mentioned that not all photons are capable of performing photoionization, and not every photoionization terminates by starting new avalanches, and, of course, not all newly-created avalanches propagate independently of the parent avalanche (some of them may be integrated into the body of the parent avalanche).

The quantity $\bar{N}$ actually represents a measure of the capability of parent avalanches to generate displaced smaller avalanches by means of the complete photoionization process. From this viewpoint, it is clear that the number of electrons adequate for this purpose must be higher than one, i.e. $\bar{N}>1$.

\subsection{Parameter J}

This parameter determines an extension of the fractal region (linear section of the graph $\log (w)$ versus $\log (n)$ ). If the whole region is measurable by the experimental device that is used, parameter $J$ can be estimated accurately and represents so many avalanche generations - i.e. "humps" [14] on the graph - that are capable of covering all the linear region measured. This is quite easy to realize, e.g., heuristically (trying various numbers of generations). In such a case, the value $J$ provides a right number of displaced avalanche generations and is equal to its upper limit $J_{\max }=d / \bar{\Delta}-1$.

However, experimental devices are sometimes not capable of measuring the whole extent of possible data. They usually measure in some restricted interval (measuring window). The result is a population distribution restricted to a certain extent which is narrower than the real extent, and parameter $J$, when fitted to the length of such a linear section, will not represent an exact number of avalanche generations but, instead, it will be smaller $J<J_{\max }=d / \bar{\Delta}-1$. This is the case for the statistics in Fig. 4 . Inserting the length $d \approx 0.7 \mathrm{~mm}$ of the discharge gap used and the value $\bar{\Delta} \approx 57.56 \mu \mathrm{m}$ into the formula of the upper limit $J_{\max }=d / \bar{\Delta}-1$, one can obtain eleven generations $J_{\max }=11$, but the fitting to the measured linear section in Fig. 4 gives $J=7<J_{\max }$, which seems to be a consequence of constrained measurements. Therefore, in both the cases - 


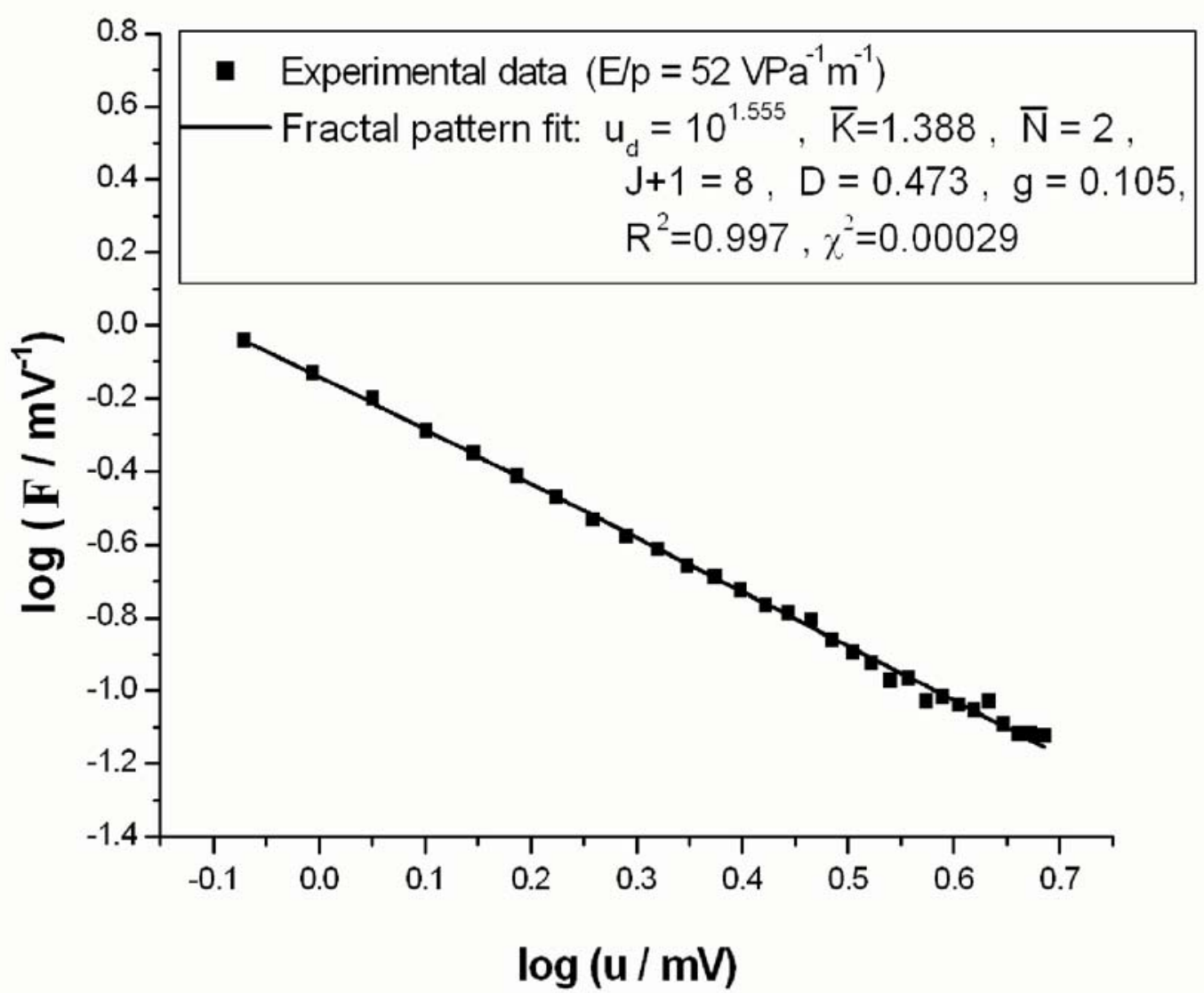

Fig. 4: Fractal pattern fit of population statistics (voltage pulses - data taken from Fig. 2). The quantity $\bar{u}_{d}=10^{1.555} \mathrm{mV}$ is a voltage scale corresponding to the average voltage pulse created by the largest avalanches detected. The quantity $g$ is not a normalization constant but only indicates the shift between unnormalized data and the unnormalized distribution $F(n, d)$. After [14].

in constrained and unconstrained measurements - the quickest way to determine $J$ is heuristic testing.

\subsection{Parameter $\bar{K}$}

Parameter $\bar{K}$, which has been termed "multiplicity", specifies multiplication (splitting) of displaced avalanches within all their generations. Its value can hardly be predicted, because the number of the displaced avalanches is a matter of purely stochastic processes, and there is no rule for estimating this quantity. However, some limitations can be specified: $\bar{K}$ cannot be zero, since in this case $\lim _{\bar{K}} D=-\infty$, which is an unacceptable result. In addition, $\bar{K} \underset{\bar{K} \rightarrow 0}{\text { must be a positive number }}$ and larger than or equal to one, i.e. $\bar{K} \geq 1$ because a fractal dimension in our case must satisfy the following relation

$$
0 \leq D=\ln (\bar{K}) / \ln (\bar{N}) \leq 3 .
$$

It is clear that the magnitudes of $\bar{K}$ and $D$ cannot be determined directly from discharge parameters $\alpha, d$ and $\bar{\Delta}$, just because $\bar{K}$ is a result of an unpredictable, stochastic process and $D$ is dependent on $\bar{K}$, i.e. $D=f(\bar{K})$. However, statistical pattern (3) has been proposed as a fitting pattern, and all its parameters, including $\bar{K}$ and $D$, can be determined either by using an optimizing procedure or heuristically. For example, if the whole distribution is measured, the entire linear section is available, from which $J_{\max }$ can be determined. As soon as $J_{\max }$ is known, the parameter $\bar{N}=\exp \left[\alpha d /\left(J_{\max }+1\right)\right]$ is available. Parameter $D$ (fractal dimension) can be estimated from the experimental data by fitting the linear section of the graph by a straight line - a regression line possessing slope $s-$ i.e., $D=-(1+s)$. Then parameter $\bar{K}$ can be estimated by us$\operatorname{ing} \bar{K}=\bar{N}^{D}$.

\subsection{Parameter $D$}

Although the fractal dimension $D$ does not explicitly occur in the statistical pattern (3), it does have a fundamental meaning. For this reason it will be convenient to discuss its physical interpretation and properties in connection with the fractal production of displaced avalanches.

The value of $D$ is defined (4) by the values of parameters $\bar{K}$ and $\bar{N}$, i.e. $D=\ln (\bar{K}) / \ln (\bar{N})$ where $\bar{K} \geq 1$ and $\bar{N}>1$. A fractal dimension, like a topological dimension, must be represented by a positive number, and because splitting of displaced avalanches occurs in the discharge gap, which is a three-dimensional Euclidean space, the fractal dimension $D$ cannot be larger than three, i.e. $0 \leq D \leq 3$. An increase in the 
$D$-value is possible only if $\bar{K}$ increases or $\bar{N}$ decreases, or if both these changes act simultaneously. An increase in $\bar{K}$ means that the "splitting" is more effective (higher multiplicity means splitting into a larger number of displaced avalanches). A decrease of $\bar{N}$ (at fixed $\alpha$ ) means a smaller critical distance $\bar{\Delta}$, and, therefore, a larger number $\left(J_{\max }\right)$ of avalanche generations $\left(J_{\max }=d / \bar{\Delta}-1\right)$, which implies a numerous set of displaced avalanches. Thus, large $D$ means that the discharge is accompanied by an abundant swarm of displaced avalanches, which can be interpreted as a tendency to delocalize the discharge over a larger portion of the inter-electrode space. In short, a higher $D$-value means a higher discharge delocalization and, conversely, a lower $D$-value indicates a more localized discharge with sporadic appearance of displaced avalanches. Since the effect of discharge localization/delocalization is undoubtedly limited, among other things by the electric field $E$ used in experiments, it will be no surprise that such a field dependence $D(E)$ has been observed previously [9], because of the acting space charges of the parent avalanches, i.e. due to the dependence $D(\alpha(E))$.

The variability of parameter $D$ when going from less populated to highly populated avalanches is well observable by comparing Figs. 4 and 5. Less populated avalanches, whose statistics are given in Fig. 4, generate lower values of dimen- sion $D$ and also smaller $N$ and $K$, in comparison with big avalanches with a prevalence of streamers (Fig. 5). This means that streamer-like avalanches split more easily into side avalanches. They are more delocalized (higher $K$ ) and are capable of filling better in the discharge gap (higher $D$ ).

\section{Fitting procedure}

Function (3), like any other multiparameter function, must be handled carefully when performing a fitting procedure. It is the starting values of the fitting parameters that have an essential influence on the results of an optimizing procedure. An inappropriate set of starting values may lead to final values that satisfy the mathematical conditions but may be physically completely unacceptable. To avoid such a failure, a proper choice of input values is necessary. In the case of function (3), there are several useful aids for establishing a proper choice.

Firstly, the value of $\bar{n}_{d}$ should be estimated directly from the statistical data rather than from the relation $\bar{n}_{d} \approx \exp (\alpha \cdot d)$, especially when the measuring device provides only a narrow acquisition range. It is suggested to estimate the $\bar{n}_{d}$ values as the horizontal asymptote of the 'lowest hump' on the graph $w(n, d)$. If only a linear section (without

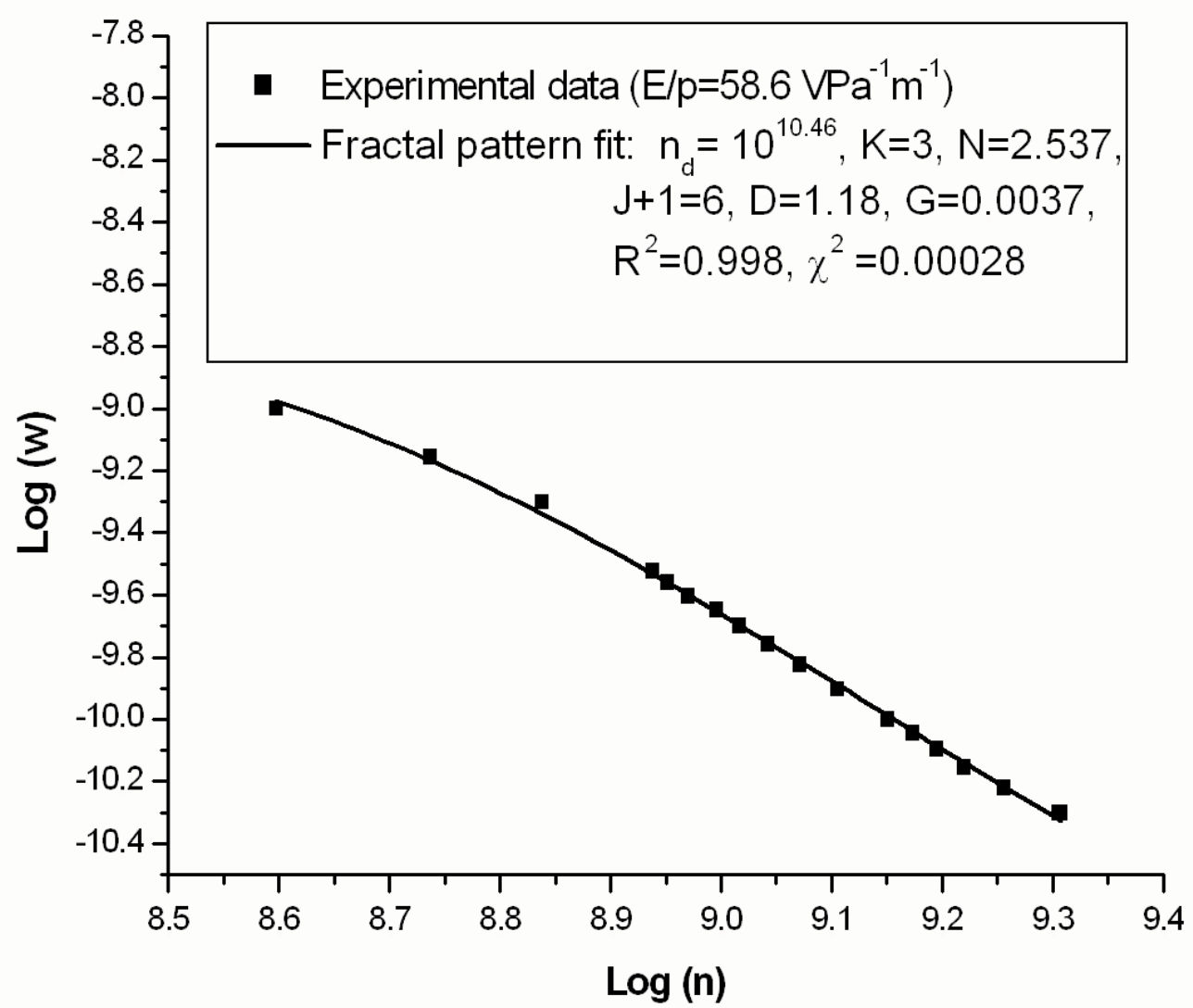

Fig. 5: Fractal population statistics of a mixture of lowly and highly populated avalanches. Data taken from Fig. 1. $G$ is a normalization constant. 
any 'humps') is available, the 'corresponding asymptote' can be simulated by using

$$
w(n, d)=\frac{1}{\bar{n}_{d}} \exp \left(-\frac{n}{\bar{n}_{d}}\right) .
$$

However, if only a restricted measuring range is available, such a value may not have the meaning of the populations of the largest parent avalanches. Instead, it may correspond to the populations of some displaced avalanches. However, from the viewpoint of the fitting procedure it will be a right choice in either case.

The second parameter $D$ can also be reliably estimated prior to starting the optimizing procedure. Linear regression of either the linear section or the 'humpy section' will provide a slope $s$ and, thus, the value $D=-(1+s)$. Parameter $D$ determines the ratio $\ln (\bar{K}) / \ln (\bar{N})$, which may assist in estimating $\bar{K}$ and $\bar{N}$ - the value of $\bar{K}$ does not usually exceed 4 .

As soon as the estimate of $\bar{n}_{d}, \bar{K}$ and $\bar{N}$ has been made, one can try to draw the graph $w(n, d)$ using only the first two terms $(J=1)$ of sum (3), and compare the result with the experimental data. After a re-adjustment of $\bar{K}$ and $\bar{N}$, further terms $(J>1)$ of sum (3) can be added until a full cover of the linear section of the measured data is accomplished. If necessary, final corrections of some parameters are recommended in order to ensure a good accord with experimental data. Only such 'pre-processed' values can be successfully used as proper input data for the chosen optimizing (fitting) procedure.

If the scenario described above is followed, it will be easier to find results that may satisfy both the mathematical and physical conditions.

\section{Transition between Furry and Pareto statistics}

The proposed statistical concept (3) is based on creating displaced avalanches formed most probably by photoionization during the initial stages of the collision process in the parent avalanches if a sufficiently high electric field (and high $\mathrm{E} / \mathrm{p}$ ) is present. The term "sufficiently high electric field" refers to such a field as is capable of ensuring the production of highly populated avalanches (for example, in air at normal atmospheric conditions $\bar{n} \geq 10^{5}$ ). In such a case, displaced avalanches may accompany the highly populated parent avalanches that terminate either without converting into streamers or as regular streamers that may or may not undergo channel branching. Therefore, the condition for the transition from Furry statistics to Pareto statistics of electron populations is a sufficiently strong electric field facilitating the creation of displaced avalanches.
There is also a simple mathematical condition ensuring the transition between these two statistics. Furry's distribution can be expected if $\bar{\Delta} \rightarrow d$. As a consequence of this condition, one can find $J_{\max }=d / \bar{\Delta}-1 \rightarrow 0$ and, in addition, $\bar{N}=\exp (\alpha \bar{\Delta}) \rightarrow \exp (\alpha d)$. This means that the parent avalanche will reach the anode without starting displaced avalanches and, thus, $\bar{K}$ loses its sense, and also $D$ cannot be rigorously defined. The condition $J_{\max }=0$ excludes the presence of displaced avalanches and, on the other hand, ensures the participation solely of parent avalanches. The parent avalanches cross the whole discharge gap and form Furry statistics (1) with an average population $\bar{n}=\exp (\alpha d)$, which represents a fixed non-fractal reference scale.

\section{Conclusion}

Instead of simple photoionization that acts solely within the primary (parent) avalanche, a new concept of displaced avalanche splitting has been proposed that allows for photoionization going beyond the parent avalanche channel, and for creating new smaller independent avalanches. The new displaced avalanches modify the overall population distribution of avalanches and cause a transition from Furry to Pareto statistics. Such a transition may occur especially when the critical distance $\bar{\Delta}$ for initiating displaced avalanches is essentially smaller than the discharge gap itself $\bar{\Delta} \ll d$. Furry and Pareto statistics can be unified into a single generalized analytical pattern that is capable of following the experimental data faithfully in both the statistical regimes (Fig 5). The main limitation of the pattern consists in its restriction to homogeneous or quasi-homogeneous background electric fields.

\section{Acknowledgments}

This work has been supported by Grant no. 202/07/1207 of the Grant Agency of the Czech Republic.

\section{References}

[1] Raether, H.: Electron Avalanches and Breakdown in Gases. London: Butterworths, 1964.

[2] Furry, W. H.: Phys. Rev., Vol. 52 (1937), p. 569.

[3] Wijsman, R.: Phys. Rev., Vol. 75 (1949), p. 833.

[4] Frommhold, L.: Zeitschrif für Physik, Vol. 150 (1958), p. 172.

[5] Richter, K.: Zeitschrift für Physik, Vol. 158 (1960), p. 312.

[6] Ficker, T.: J. Appl. Phys., Vol. 78 (1995), p. 5289.

[7] Ficker, T., Macur, J., Kliment, M., Filip, S., Pazdera, L.: J. El. Eng., Vol. 51 (2000), p. 240.

[8] Ficker, T., Macur, J., Pazdera, L., Kliment, M., Filip, S.: IEEE Trans. Diel. El. Insul., Vol. 8 (2001), p. 220. 
[9] Ficker, T.: IEEE Trans. Diel. El. Insul., Vol. 10 (2003), p. 689.

[10] Ficker, T.: IEEE Trans. Diel. El. Insul., Vol. 10 (2003), p. 700 .

[11] Ficker, T., Macur, J., Kapička, V.: Czech. J. Phys., Vol. 53 (2003), p. 509.

[12] Ficker, T.: IEEE Trans. Diel. El. Insul., Vol. 11 (2004), p. 136.

[13] Mandelbrot, B. B.: The Fractal Geometry of Nature. New York: Freeman, 1983.

[14] Ficker, T.:J. Phys. D: Appl. Phys., Vol. 40 (2007), p. 7720.

[15] Kulikovski, A. A.: J. Phys. D: Appl. Phys., Vol. 33 (2000), p. 1514.

[16] Pancheshnyi, S.: Plasma Sour. Sci. Technol., Vol. 14 (2005), p. 645.

[17] Arrayas, M., Ebert, U., Hundsdorfer, W.: Phys. Rev. Lett., Vol. 88 (1998), p. 174502.
[18] Montijn, C., Ebert, U., Hundsdorfer, W.: Phys. Rev. E, Vol. 73 (2006), p. 065401-1.

[19] Penney, G. W., Hummert, G. T.: J. Appl. Phys., Vol. 41 (1970), p. 572.

Prof. RNDr. Tomáš Ficker, DrSc.

Phone: +420 541147661

e-mail: ficker.t@fce.vutbr.cz

Department of Physics

Faculty of Civil Engineering

Brno University of Technology

Veveří 95

66237 Brno, Czech Republic 